\title{
Stereoscopic Static Depth Perception of Enclosed 3D Objects
}

\author{
Filipe M. M. Marreiros ${ }^{1,2 *}$ \\ Örjan Smedby ${ }^{1,2,3 \dagger}$ \\ ${ }^{1}$ Center for Medical Image Science and Visualization (CMIV) \\ ${ }^{2}$ Department of Science and Technology (ITN) - Media and Information Technology (MIT) \\ ${ }^{3}$ Department of Radiology (IMH) \\ Linköping University, Sweden
}
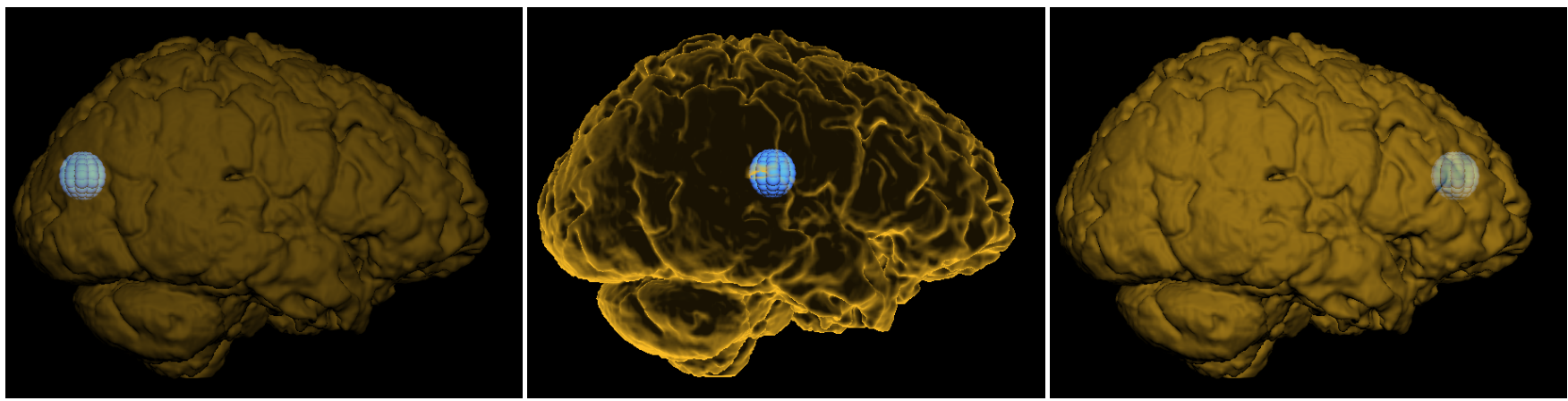

Figure 1: Lateral view of the positions where the enclosed object (simulated tumor) is located within the exterior object (brain). Back position (left), center position(middle), front position(right). Two rendering methods were used with different opacity values.

\section{Abstract}

Depth perception of semi-transparent virtual objects and the visualization of their spatial layout are crucial in many applications, in particular medical applications. Depth cues for opaque objects have been extensively studied, but this is not the case for stereoscopic semi-transparent objects, in particular in the case when one $3 \mathrm{D}$ object is enclosed within a larger exterior object.

In this work we explored different stereoscopic rendering methods to analyze their impact on depth perception accuracy of an enclosed 3D object. Two experiments were performed: the first tested the hypotheses that depth perception is dependent on the color blending of objects (opacity - alpha) for each rendering method and that one of two rendering methods used is superior. The second experiment was performed to corroborate the results of the first experiment and to test an extra hypothesis: is depth perception improved if an auxiliary object that provides a relationship between the enclosed object and the exterior is used?

The first rendering method used is simple alpha blending with Blinn-Phong shading model, where a segmented brain (exterior object) and a synthetic tumor (enclosed object) were blended. The second rendering method also uses Blinn-Phong, but the shading was modified to preserve silhouettes and to provide an illustrative rendering. Comparing both rendering methods, the brighter regions of the first rendering method will become more transparent in the second rendering method, thus preserving silhouette areas.

The results show that depth perception accuracy of an enclosed object rendered with a stereoscopic system is dependent on opacity for some rendering methods (simple alpha blending), but this effect is less pronounced than the dependence on object position in relation to the exterior object. The illustrative rendering method is less dependent on opacity. The different rendering methods also perform slightly differently; an illustrative rendering method was

*e-mail:filipe.marreiros@liu.se

†e-mail:orjan.smedby@liu.se superior and the use of an auxiliary object seems to facilitate depth perception.

CR Categories: I.3.7 [Computer Graphics]: Three-Dimensional Graphics and RealismVirtual Reality-; I.4.8 [Image Processing and Computer Vision]: Scene Analysis-Depth cues; I.4.8 [Image Processing and Computer Vision]: Scene Analysis-Object recognition;

Keywords: depth perception, stereoscopy, enclosed 3D objects

\section{Introduction}

The correct depth perception of semi-transparent virtual objects and the visualization of their spatial layout are crucial in many applications. Medical applications are a good example where accurate visualization is required in particular if surgical procedures are dependent on the visualization. Sielhorst et al. [Sielhorst et al. 2008] and Bianchi [Bianchi 2006] present several surgical augmented reality visualization systems.

Extensive literature regarding depth perception and shape perception exists. For depth perception the several depth cues, their strengths and relationships are explored [Cutting and Vishton 1995], but in general they are used considering opaque objects. Regarding shape perception we can also find specific cues [Todd 2004] and some of them like motion are common in both cases. There is a clear interdependency between depth and shape.

Perception of transparent 3D objects has been addressed in some

Permission to make digital or hard copies of part or all of this work for personal or classroom use is granted without fee provided that copies are not made or distributed for commercial advantage and that copies bear this notice and the full citation on the first page. Copyrights for components of this work owned by others than ACM must be honored. Abstracting with credit is permitted. To copy otherwise, to republish, to post on servers, or to redistribute to lists, requires prior specific permission and/or a fee. Request permissions from permissions@acm.org.

SAP 2013, August 22 - 23, 2013, Dublin, Ireland.

Copyright (C) ACM 978-1-4503-2262-1/13/08 \$15.00 
studies. Boucheny et al. [Boucheny et al. 2009] present a perceptive evaluation of volume rendering techniques. They used Direct Volume Rendering (DVR) techniques, which are commonly used for the visualization of semi-transparent medical data where different tissue types are blended together. In their work, the participants were asked to decide how two semi-transparent cylinders are organized in depth, with a small intersection region on the rendered image. The cylinders have different widths and luminance values. They report that the participants have poor results due to the relative size and luminosity cues. Interrante et al. [Interrante et al. 1997] address transparency of enclosed objects to convey 3D shape via texture. Nakayama et al. [Nakayama et al. 1990] analyze transparency in relation to depth, subjective contours, luminance, and neon color spreading. Kersten et al. [Kersten et al. 2006] explore the enhancement of depth perception in translucent volumes. A perception-based transparency optimization for DVR is presented by Chan et al. [Chan et al. 2009] and perceptually based depthordering enhancement for DVR by Zheng et al. [Zheng et al. 2013].

Perception of transparency using stereoscopic systems can be found in [Akerstrom and Todd 1988] where the transparency of overlapping surfaces using random-dot stereograms is investigated and in [Tsirlin et al. 2008] that investigates the constraints on the perception of multiple surfaces also using dot stereograms. Maurer et al. [Maurer et al. 2001] used stereoscopic augmented reality systems to visualize brain structures inside a head phantom with different rendering methods. Since the user can move around the phantom, kinetic cues are also present. Sielhorst et al. [Sielhorst et al. 2006] developed another augmented reality system where surgeons are asked to point at specific locations using a pointer also with multiple rendering methods. In both previous studies it was reported that the results are considerably different for the several rendering methods.

Although the depth cues for opaque objects have been extensively studied, this is not the case for stereoscopic semi-transparent objects, in particular in the case when one $3 \mathrm{D}$ object is enclosed within a larger exterior object. To our knowledge, there are no studies that analyze the dependency of depth perception accuracy on color blending and position of the enclosed object rendered using a stereoscopic display system. To some extent Maurer et al. [Maurer et al. 2001] and Sielhorst et al. [Sielhorst et al. 2006] explored different stereoscopic rendering methods (in a medical context) to analyze their impact on depth perception accuracy. Here we will study alternative rendering methods that preserve the shape of both enclosed and exterior object.

The aim of this study is to explore these unknowns, i.e. how, in a stereoscopic set-up, depth perception accuracy depends on color blending (opacity), position of an enclosed object and rendering method. The first hypothesis to be tested was that depth perception is dependent on the color blending of objects (opacity - alpha) and that one of two rendering methods used is superior. The second hypothesis states that depth perception is improved if an auxiliary object providing a relationship between the enclosed object and the exterior is used. We restrict our attention to the visualization of enclosed objects, i.e. objects that are totally within a larger exterior object. Ultimately we want to use our system for the visualization of medical datasets, in particular tumors located in the brain.

\section{Material and methods}

\subsection{Apparatus}

This work requires a stereoscopic display system to present the stimuli. A solution where no ghosting artifacts are present is needed as this effect degrades considerably stereoscopy. Few stereoscopic displays are totally ghosting-free. One such system, proposed originally by Hart [Hart ], [Boháč ] consists of a dual-monitor singlemirror where the viewer has to place his eyes at a certain angle between the edge of the mirror. The system was modified in order to use only one monitor and one mirror, and to allow a straight view (as opposed to angled). The geometry for the reflected image needs special treatment and relies on the reflection matrix introduced by Bimber et al. [Bimber et al. 2000], [Bimber et al. 2001]. Since we are using DVR, we can compute the geometry per ray and generate an accurate image.

\subsection{General stimuli}

The two experiments in the following sections use the same medical dataset. It consists of an approximately isotropic 3D T1-weighted Magnetic Resonance (MR) head image acquired at 1.5 T, where the brain has been segmented using a threshold-based level set method reported in [Wang et al. 2011]. A binary volume mask is generated to exclude regions outside the brain. This dataset was used since our main goal is to identify structures inside the brain, e.g. tumors. It also has a somewhat irregular shape which may increase the difficulty to perceive depth relationships.

The enclosed object represents a brain tumor. It consists of a group of small spheres connected together in a sphere-like fashion. Three depth positions were selected for the tumor location as can be seen in the lateral views presented in Fig. 1 (the experimental stimuli are presented in a frontal view). We named them: back (posterior), center, front (anterior). The tumor is always located inside the right hemisphere of the brain.

\subsection{Participants}

The majority of the participants were students of Linköping University. To all participants an informed consent form was provided, where a description of the experiments and their purpose was given. A stereo blindness test was also performed using an anaglyph image [Schwartz 2003], where the users were asked to reply the number seen hovering the foreground. A small number of participants (3 in experiment 1 and 2 in experiment 2 ) had difficulties in replying, but once using the stereoscopic system they could see the brain with a good depth sensation, and for this reason we did not exclude them.

In addition, lateral view images to exemplify the location of the inner and outer objects at different depth locations were provided before the experiments to aid the participants. In Fig. 1, the positions are presented with the two different rendering methods used to familiarize the participants with the stimuli.

\subsection{Rendering methods}

Two rendering methods were used for the experiments. Both use the Blinn-Phong shading model [Blinn 1977], but only the diffuse component. Initially the colors for the brain and tumor are computed then they are mixed to produce the final pixel color. The color blending is performed using alpha blending. The second rendering method requires the computation of view-dependent intermediate alpha and color values.

In the first rendering method (simple alpha blending), the final color is obtained according to the alpha blending, Equation 1.

$$
c \text { Final }=c \text { Brain } \times(1.0-\text { alpha })+c \text { Tumor } \times \text { alpha }
$$

The second rendering method (illustrative) uses angle-based opacity [Carnecky et al. 2013]. It produces results similar to illustrative rendering where more emphasis is given to regions where the surface normal is pointing away from the viewer, or where the dot 
product of the surface normal and the viewing direction is larger [Salah et al. 2005] (silhouette extraction).

In this case, a slight variation was performed; instead of the viewing direction the Blinn-Phong Half vector was used. The results are similar, but proceeding this way the values of the brain BlinnPhong shading can be used directly (the dot product is computed only once) for color and opacity (alpha) Equations 2 and 3.

$$
c \text { Brain }^{\prime}=\operatorname{dot} P(\text { halfVector, } \operatorname{surfNormal}) \times c \text { Brain }
$$

$$
\text { alpha' }=\operatorname{dot} P(\text { half Vector }, \text { surf Normal }) \times \text { alpha }
$$

Then alpha blending is performed to obtain the final result.

$$
c \text { Final }=c \text { Brain }^{\prime} \times\left(1.0-\text { alpha }{ }^{\prime}\right)+c \text { Tumor } \times a l p h a^{\prime}
$$

Comparing both rendering methods, the brighter regions of the first rendering method will become more transparent in the second rendering method, thus preserving silhouette areas.

The colors of the brain and the tumor are opposite and have the same luminance value, providing good contrast.

\subsection{Experiment 1}

In this experiment two hypotheses were tested:

- the depth perception is dependent on the color blending of objects (opacity-alpha) per rendering method

- one of two tested rendering methods is superior

The independent variables in this experiment are the rendering methods $(\mathrm{RM} 1=$ simple alpha blending, $\mathrm{RM} 2=$ illustrative $)$, the opacity $($ alphaRM1 $=\{0.2,0.3,0.4,0.5,0.6,0.7,0.8\}$, alphaRM2 $=\{0.6,0.7,0.8,0.9,1.0,1.1,1.2\})$ and the position of the enclosed object (back, center, front) the dependent variable is the correctness of the perceived position (correct, incorrect). Please note that the range of alpha values for the illustrative rendering is higher these values were selected in order to preserve structural information. The total number of permutations is: $2 \times 7 \times 3=42$. A total of 42 participants underwent the experiment, 9 females and 33 males with an average age of 33.33 years. The youngest participant age was 24 and the oldest, 59.

\subsubsection{Stimuli and procedure}

The stimuli presented to the subjects consist of the encoded object (representing the tumor), the brain and a dotted pattern in the background (Fig. 2). The red line indicates where the mirror is placed in order to obtain a stereoscopic visualization. The image on the right is skewed and vertically flipped due to the reflection on the mirror. By directly looking at the mirror, the user perceives a similar image as in the left (with appropriate disparity). The background dots are used to aid the perception of transparency as reported in Johnson et al. [Johnson and Aslin 2000].

Each participant was asked to answer where he perceives the enclosed object (back, center, front or cannot tell) 42 times corresponding to the total amount of permutations. These answers were checked against the real positions to verify their correctness. In the cases when the participant could not tell, this was considered as an incorrect position. A latin random square $42 \times 42$ matrix was used.

\subsection{Experiment 2}

This experiment is intended to improve the design and corroborate the results of experiment 1 . Particularly the relative size effect of the enclosed object is minimized by using different sizes at the different positions. The common results can be compared to experiment 1 to check for consistency.

In addition we wanted to explore another hypothesis: is depth perception improved if an auxiliary object that provides a relationship between the enclosed object and the exterior is used?

The independent variables in this experiment are the rendering method, auxiliary object, and the position of the enclosed object (back, center, front) the dependent variable is the correctness of the perceived position (correct, incorrect). 3 different sizes per position of the enclosed object were used. The total number of permutations is: $2 \times 2 \times 3 \times 3=36$. A total of 36 participants underwent the experiment, 15 females and 21 males with average age of 36.47 years. The youngest participant age was 26 and the oldest, 59 .

\subsubsection{Stimuli and procedure}

The stimuli and procedure were similar to experiment 1 . The main differences are that fixed opacities for the rendering methods were used. For each rendering method, two tests were performed, with and without the auxiliary object, and 3 different sizes per position were used. In order to minimize image size differences of the projected enclosed object we needed to transform the original 3D enclosed object size $\left(s i z e_{0}\right)$ at each position to approximately match the projected sizes of all positions:

$$
\begin{aligned}
& \text { - front }\left\{\text { size }_{0}-2 \beta, \text { size }_{0}-\beta,\right. \text { size } \\
& \text { - } \operatorname{center}\left\{\text { size }_{0}-\beta, \text { size }_{0}, \text { size }_{0}+\beta\right\} \\
& \text { - } \operatorname{back}\left\{\text { size }_{0}, \text { size }_{0}+\beta, \text { size }_{0}+2 \beta\right\}
\end{aligned}
$$

These operations will generate for all positions 3 projected size approximations to the original projections of the front, center and back positions. $\beta$ is a factor used in order to minimize the projected size differences, for example: considering the front position, to approximate the projected size of the center position, the $3 \mathrm{D}$ enclosed object size $\left(\right.$ size $\left._{0}\right)$ must be decreased by $\beta$ and to approximate the projected size of the back position an even stronger reduction is required $2 \beta$. The same reasoning is performed for the remaining positions. $\beta$ was chosen so that the size transformations are consistent between positions.

The auxiliary object consists of a vertical dotted line that crosses the center of the tumor and starts and ends outside the brain as presented in Fig. 3. A vertical line was used because it is perpendicular to the disparity direction.

A different background was used with a non-regular pattern in the horizontal direction with dots of different sizes. In the previous experiment, the dots were equal, and this can lead to ambiguities, so that the observer tries to merge the wrong match.

For both rendering methods the same opacity value was used: alpha $=0.62$, a value close to the optimal opacity value found for rendering method 1 (see below). To calculate alpha' for the illustrative rendering method, Equation 3 was used.

Fig. 3 presents the stimuli for the illustrative rendering method and the simple alpha blending rendering method, both using an auxiliary object. 


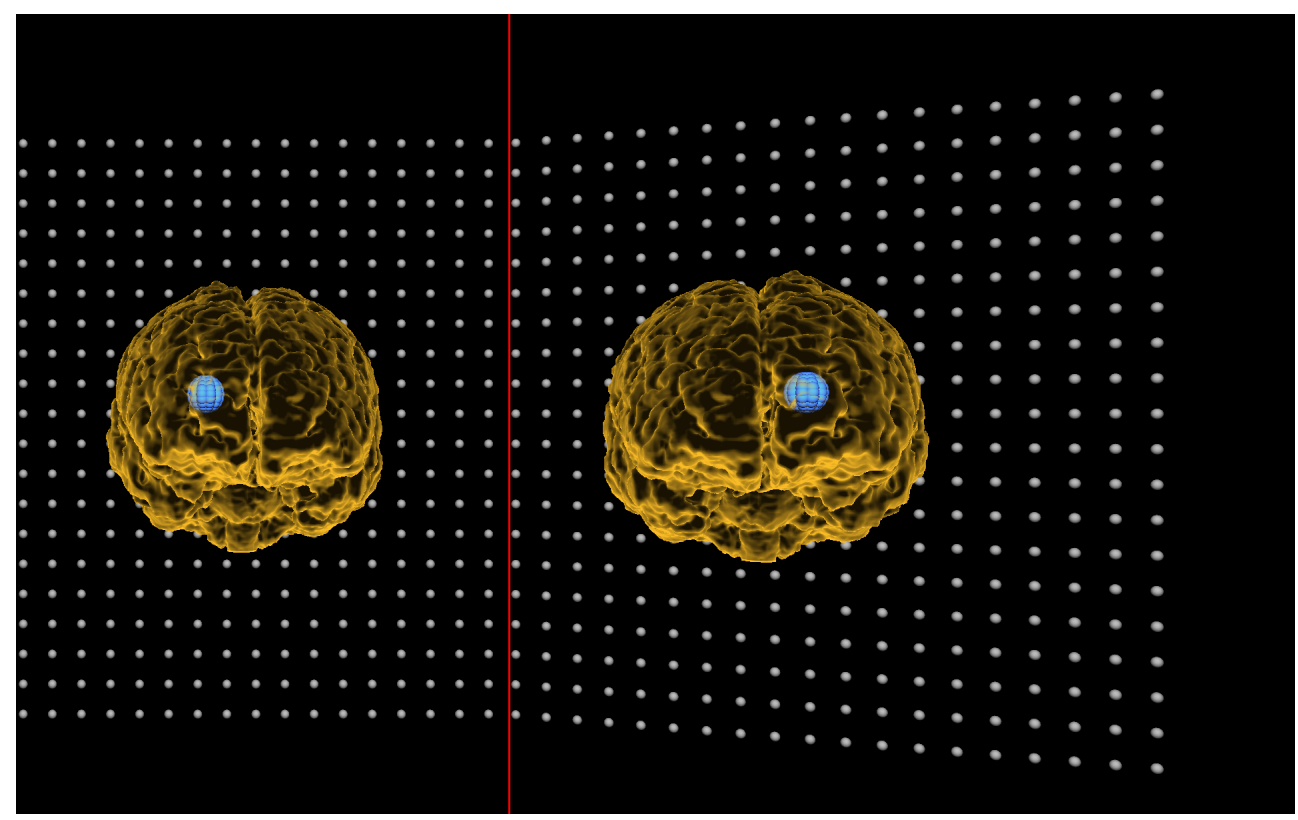

Figure 2: Stereo pair used in experiment 1.

\subsection{Statistical methods}

For both experiments, the accuracy of the answers was analyzed with a mixed-effects binary logistic regression model.

In experiment 1 , the simple alpha blending rendering method and the illustrative rendering method were analyzed both together and separately, treating participant identity as a random effect (since the participants are not interesting in themselves but just represent a sample from a larger population). The fixed effects were position, viewing order, (normalized) opacity and (when comparing methods) rendering method. For opacity, a linear or a quadratic dependence was used according to the significance level of the seconddegree term. From the quadratic equation, an optimal opacity value was calculated, maximizing the predicted probability of a correct answer, together with its $95 \%$ confidence interval.

In experiment 2 , both rendering methods were analyzed together. Participant was again treated as a random effect. The fixed effects were position, rendering method, auxiliary object and viewing order.

All statistical calculations were made in Stata 12.0 (StataCorp, College Station, TX, USA), and the limit for significance was set to $p=0.05$.

\section{Results}

\subsection{Experiment 1}

The results of experiment 1 are summarized in Fig. 4. The difference between higher accuracy values for the illustrative rendering method than for the simple alpha blending method, which is visually obvious in the Figure, was significant $(p<0.001)$ when the opacity values were normalized, so that they covered the same interval for both methods, but not when non-normalized values were used $(p>0.20)$, so that numerically identical opacity values were compared with each other.

When all observations for rendering method 1 were analyzed in one logistic regression model, there was a significant quadratic dependency on opacity $(p<0.01)$, and both the back position and the center position had significantly $(p<0.001)$ lower accuracy than the front position. The difference between the center and the back position was not significant. In addition, there was a significant $(p<0.001)$ increase in accuracy with viewing order, suggesting that learning effects are present. This can also be observed in Fig. 5, where the accuracy of all possible viewing order values is presented, and it is seen that the values are slightly lower in the beginning of the viewing session. (The prediction curve is not a straight line, since the logistic regression model involves a nonlinear logit transformation.) The optimal opacity value predicted from the quadratic relationship was 0.64 , with the $95 \%$ confidence limits 0.53 and 0.75 (Fig. 6).

When analyzing individual positions of the simple alpha blending rendering method, for the front position, the quadratic model was not appropriate, but there was a significant $(p<0.01)$ increase in accuracy with increasing opacity, with no significant effect of viewing order. For the center position, the quadratic model was appropriate, and there was a significant quadratic dependence on opacity $(p<0.05)$. In addition, there was a significant $(p<0.001)$ increase in accuracy with viewing order, suggesting that learning effects are present. For the back position, too, there was a significant quadratic dependence on opacity $(p<0.01)$ but no significant dependence on viewing order.

The graphical representation of the appropriate models and the values observed can be seen in Fig. 7.

For the illustrative rendering method, there was no significant effect of opacity (Fig. 4, right), but both the back position and the center position had significantly $(p<0.001)$ lower accuracy than the front position. A significant dependence was also present for viewing order $(p<0.001)$, suggesting that learning effects are present.

\subsection{Experiment 2}

When all observations were statistically analyzed, using a linear model, the simple alpha blending rendering method gave signifi- 


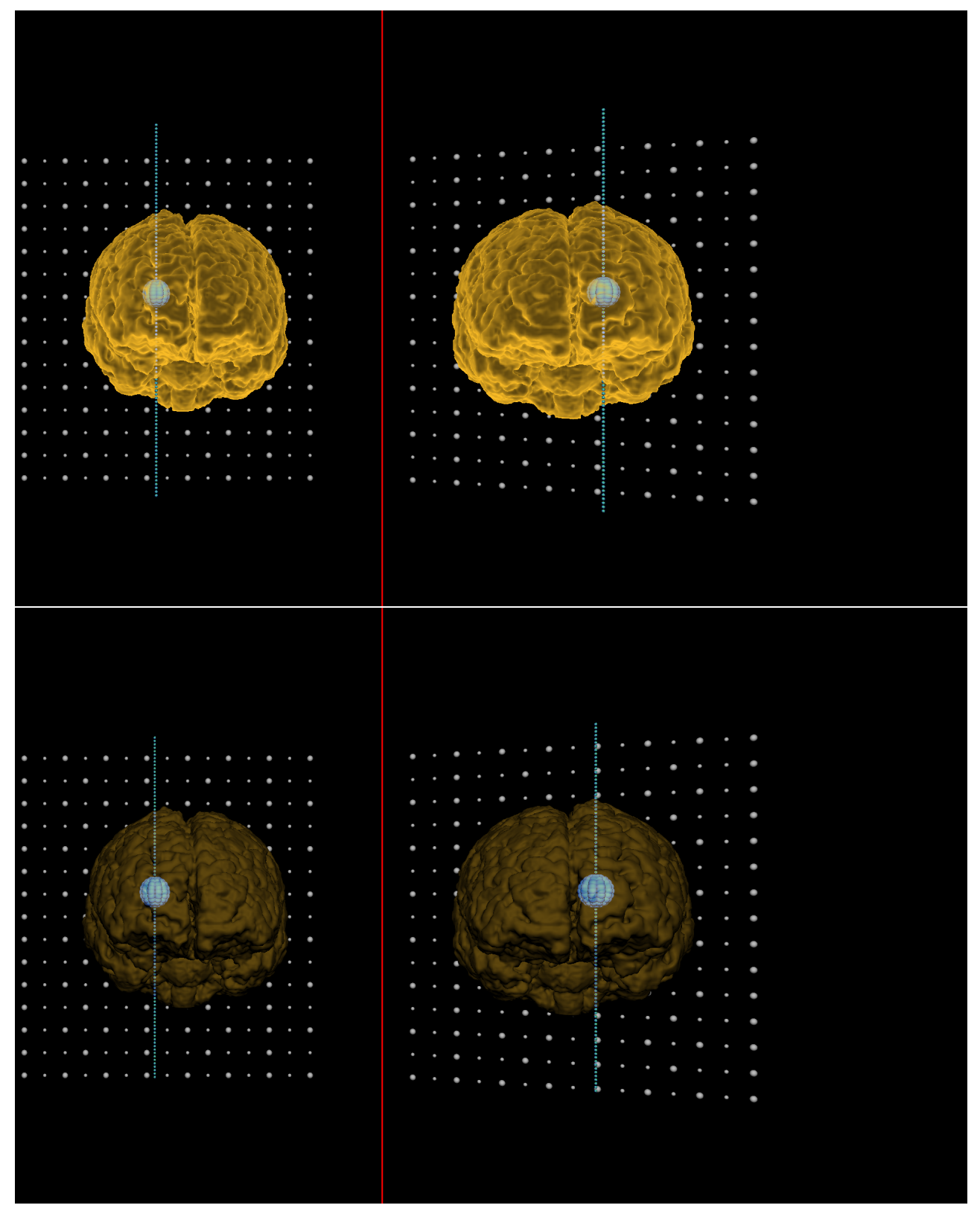

Figure 3: Stereo pair used in experiment 2. Illustrative rendering method (top) and simple alpha blending rendering method (bottom), both using the auxiliary object (dotted line).
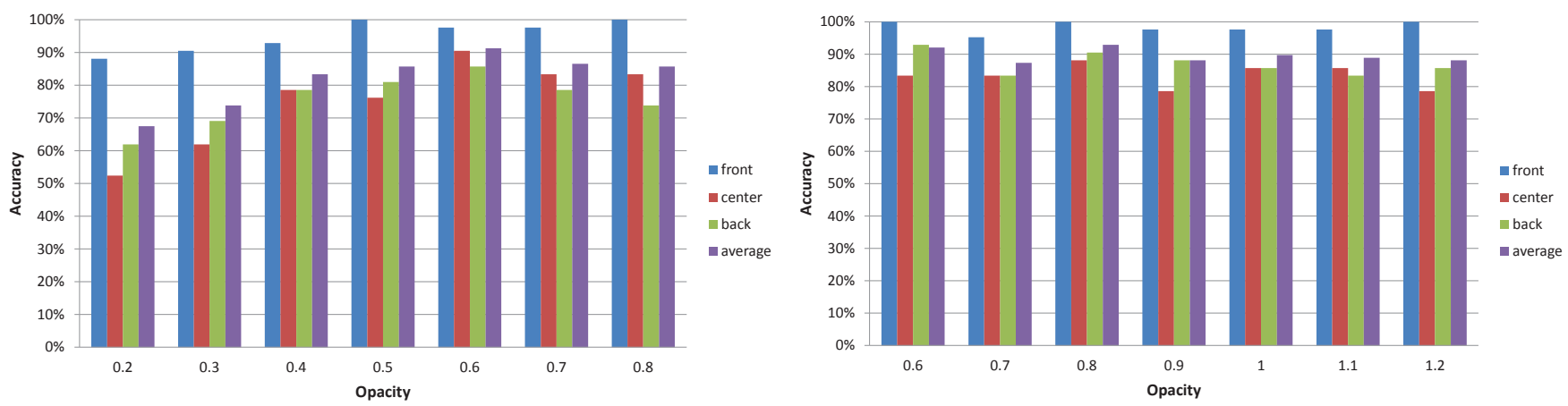

Figure 4: Accuracy values in experiment 1 for each position and alpha value. Simple alpha blending rendering method (left) has significantly $(p<0.001)$ lower accuracy than the illustrative method (right). 


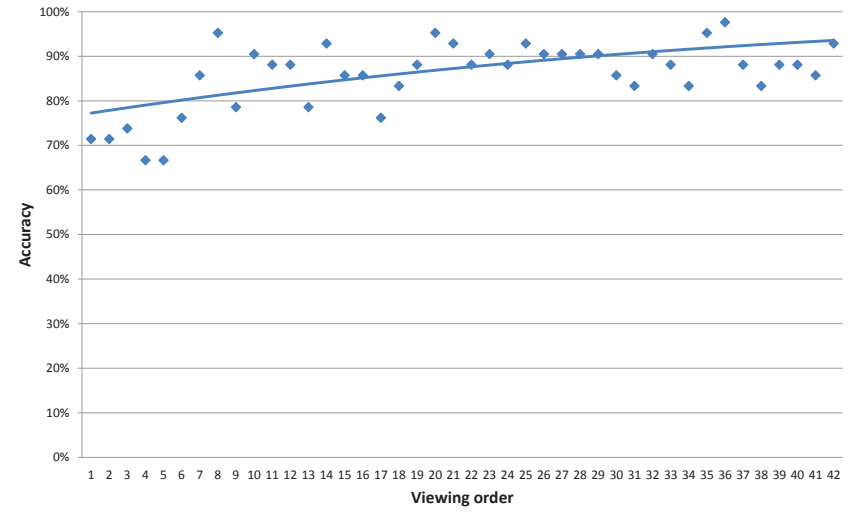

Figure 5: Accuracy of all observed values of viewing order. Values predicted by the statistical model indicated by curve. Accuracy increases significantly $(p<0.001)$ with viewing order.

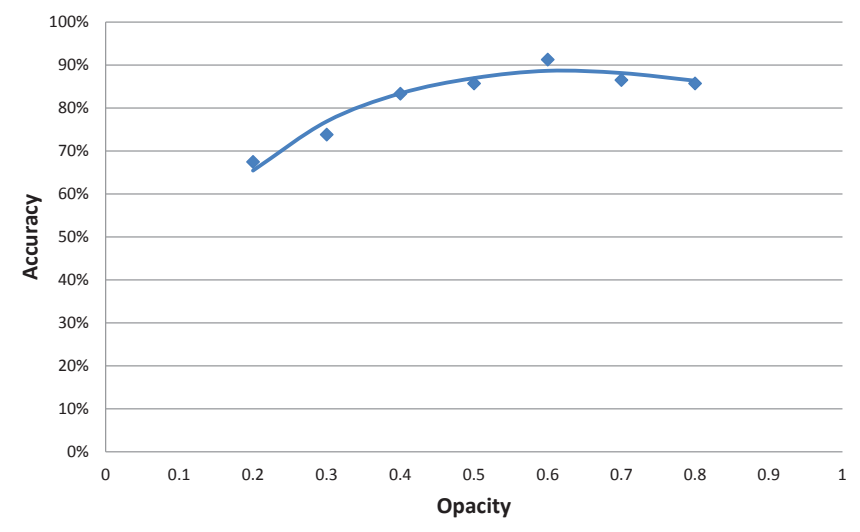

Figure 6: Quadratic model fit (curve) $(p<0.01)$ and observed accuracy values (dots) of all the observations, simple alpha blending rendering method.

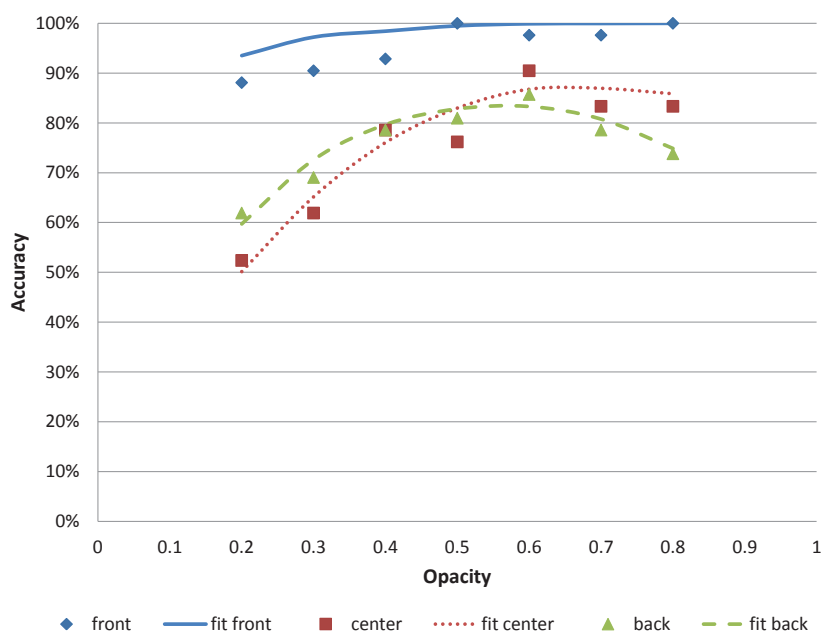

Figure 7: Quadratic model fit of for center $(p<0.05)$ and back $(p<0.01)$ positions and linear model fit for the front position ( $p<0.01)$ (curves) and observed values (dots) for all positions of the simple alpha blending rendering method.

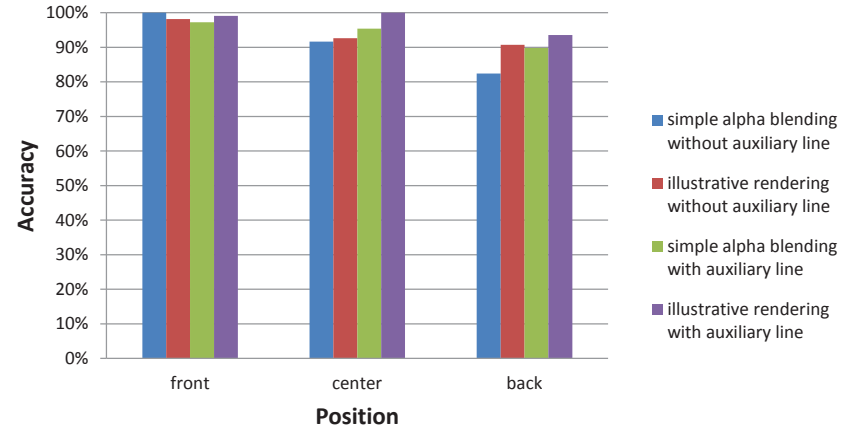

Figure 8: Accuracy in experiment 2 for different positions and different rendering methods.

cantly ( $p<0.01$ ) inferior results compared to the illustrative rendering method (with and without auxiliary object) (Fig. 8).

Significantly ( $p<0.01$ ) better results were found if the auxiliary object was used. The front position was significantly $(p<0.01)$ better than both the center and back positions, and the back position was significantly $(p<0.01)$ worse than the center position. No significant effect of viewing order was found.

\section{Discussion and conclusion}

The results show that the illustrative rendering method was superior to the simple alpha blending rendering method for depth perception position accuracy of enclosed objects visualized using a stereoscopic system. Although both methods use the Blinn-Phong shading model, the shading of the illustrative rendering method was modified to preserve the silhouette areas (shape information) and deemphasize the remaining areas. These modifications impacted significantly the performance of the rendering method as discussed below.

The motivation behind the use of the selected rendering methods is their simplicity allowing iterative frame rates and their ability to preserve to some extent important shape features that may relate to anatomical locations (considering the brain - gyri and sulci) used by surgeons for navigation purposes. These important features may be deemphasized if other visualization methods using points or wireframe are considered.

The results from experiment 1 show that for the simple alpha blending rendering method, depth perception depends on opacity. In the center and back positions, this dependence is stronger than in the front. In fact, for the front position the quadratic model was unable to fit the data. For the front, within the range studied, all values were above $88 \%$, i.e. approximately the same as the highest value of the center or back positions. This indicates that position has a stronger influence then opacity in depth perception. But if the enclosed object is at a certain depth position (not the front position), then the dependence on opacity is stronger. Considering all positions, the optimal opacity value seems to be around 0.6. It should be pointed out that a quadratic model was not expected a priori, but since the appearance of several of the graphs indicated that there is an optimum at an intermediate opacity value rather than at one of the extremes, we wanted to apply a model that allows for such an optimum. The simplest model fulfilling this requirement includes a quadratic equation.

For the illustrative rendering method, the results indicate that there is no relation between depth perception and opacity in the opacity range tested. The front position is significantly better then the re- 
maining. But it is not clear whether there is a linear dependency of depth perception accuracy and the position of the enclosed object.

A significant difference in accuracy for both rendering methods was found when using normalized opacity values. The original opacities (alpha) generate non-comparable representations for both rendering methods due to the extra transformations of the illustrative rendering method, (Equations 2, 3). For this reason the normalization was required in order to study the same range of values. This difference was further studied in experiment 2 using a constant optimal opacity value for both rendering methods (see below).

With both rendering methods, a significant learning effect was also found. This might be partially due to one confounding factor in experiment 1 . This factor is relative size since it is also a depth cue. The enclosed object is rendered always with the same 3D size which will lead to a smaller image size for the positions further away. Although there was a time delay between the stimuli, it is possible that the user memorized these size differences.

Comparing the results of experiment 1 and 2 for each rendering method, without the auxiliary object, we found values in the same range. This indicates that relative size did not have a major impact in the results of experiment 1 .

The results of experiment 2 indicate that the use of an auxiliary object increases depth perception accuracy. The differences between rendering methods are significant, with the illustrative rendering method providing providing better accuracy. These relationships are cleared for positions further away. If the enclosed object is placed near the surface of the exterior object then there is no need to use the auxiliary object and either of the rendering methods is appropriate. No significant learning effect was found in this experiment.

There are some relevant earlier results comparing rendering methods, [Maurer et al. 2001] reports that if the enclosed object is rendered with wire-frame or texture-mapped dot patterns, the depth perception accuracy increases. These illustrative rendering methods are closely related to our illustrative rendering method, which also provided better accuracy in our experiments. The difference is that in our case the object rendered with the illustrative method was the exterior object, not the enclosed object. It seems that illustrative rendering methods provide added value in depth perception accuracy similar results were found for shape where the exterior object was enhanced with texture [Interrante et al. 1997].

With the simple alpha blending rendering method, it was noticed that the optimal opacity value was higher for the enclosed object $(0.62)$ than for the exterior object $(1-0.62)$. Since we are using the same values of the base color luminance, this implies that the luminance of each surface is mainly dependent on opacity (shading also plays a role). In the optimal case, the luminance value is higher for the enclosed object then for the exterior surface. Traditionally for monoscopic renderings using perception models, the reverse relation is used to aid in depth ordering, i.e. the closer surface to the observer has a higher luminance [Zheng et al. 2013]. This is a clearly ambiguous phenomenon that should be further studied.

Although our analysis was designed to focus only on the relationships between the factors under study and depth perception accuracy, there might still exist confounding factors not accounted for. Shape can be one of them, although an attempt was made to provide complex shapes for the analysis. The correct head position of the participant may also slightly influence the results. Considering the strong dependencies found, we believe that these confounding factors, if present, are minimal.

Alternative solutions to treat the blending could be considered, for instance we could perform a compositing of all brain surface inter- ceptions until the enclosed volume is hit or the ray exits the brain. This could provide some extra information about the boundaries of the brain. But how the overall compositing of the objects can be accurately performed is not clear. In addition we could also consider the background color in the compositing, e.g. to provide a white background.

In the future we want to study depth perception accuracy with more than two objects (or two surface intersections) and not only limited to one enclosed object. This relates to the more general situation found in DVR. Ultimately we want to find guidelines for stereoscopic DVR in order to obtain high depth perception accuracy.

In conclusion this study shows that depth perception accuracy of an enclosed object rendered with a stereoscopic system is dependent on opacity for some rendering methods (simple alpha blending), but this effect is less pronounced than the dependence on object position in relation to the exterior object. The different rendering methods also perform slightly differently; an illustrative rendering method was superior and the use of an auxiliary object seems to facilitate depth perception.

\section{Acknowledgements}

This work was funded by the Visualization programme of the Swedish Foundation for Strategic Research, the KK Foundation, Vinnova, Invest in Sweden Agency, Vrdalstiftelsen (grant 2009/0079) and the Swedish Childhood Cancer Foundation (grant no. MT2013-0036). The authors are indebted to all the participants of the study for their time and commitment that made this work possible.

\section{References}

Akerstrom, R. A., And TodD, J. T. 1988. The perception of stereoscopic transparency. Perception \& Psychophysics 44, 5, 421-432.

BIANCHI, G. 2006. Exploration of Augmented Reality Technology for Surgical Training Simulators. PhD thesis, Swiss Federal Institute of Technology Zurich.

Bimber, O., Encarnação, L. M., And Schmalstieg, D. 2000. Augmented reality with back-projection systems using transflective surfaces. Computer Graphics Forum (Proceedings of EUROGRAPHICS 2000) 19, 3, 161-168.

Bimber, O., Fröhlich, B., Schmalstieg, D., AND ENCARNAÇÃO, L. M. 2001. The virtual showcase. IEEE Computer Graphics and Applications 21, 6, 48-55.

BLINN, J. F. 1977. Models of light reflection for computer synthesized pictures. ACM SIGGRAPH Computer Graphics 11, 2 , 192-198.

BонÁč, M. Dual monitor set-up for stereoscopic viewing. http://klub.stereofotograf.eu/dual_monitor.php.

Boucheny, C., Bonneau, G., Droulez, J., Thibault, G., AND PLOIX, S. 2009. A perceptive evaluation of volume rendering techniques. ACM Transactions on Applied Perception (TAP) 5, 4 (January).

CARnecky, R., Fuchs, R., Mehl, S., JAng, Y., AND Peikert, R. 2013. Smart transparency for illustrative visualization of complex flow surfaces. IEEE Transactions on Visualization and Computer Graphics 19, 5 (May), 838-851.

Chan, M., Wu, Y., MaK, W., Chen, W., And QU, H. 2009. Perception-based transparency optimization for direct volume 
rendering. IEEE Transactions on Visualization and Computer Graphics 15, 6 (November/December), 1283-1290.

Cutting, J. E., And Vishton, P. M., 1995. Perceiving layout and knowing distances: The integration, relative potency, and contextual use of different information about depth. In W.Epstein and S. Rogers (Eds.), Perception of space and motion, New York: Accademic Press.

HART, J. Dual monitor digital viewing. http://www.crystalcanyons.net/abouts/3dc9_DualMonitorComplete.shtm.

Interrante, V., Fuchs, H., And Pizer, S. M. 1997. Conveying the $3 \mathrm{~d}$ shape of smoothly curving transparent surfaces via texture. IEEE Transactions on Visualization and Computer Graphics 3, 2 (April/June), 98-117.

Johnson, S. P., AND Aslin, R. N. 2000. Infants' perception of transparency. Developmental Psychology 36, 6, 808-816.

Kersten, M. A., Stewart, A. J., Troje, N., And Ellis, R. 2006. Enhancing depth perception in translucent volumes. IEEE Transactions on Visualization and Computer Graphics 12, 5 (September/October), 1117-1123.

Maurer, C. R., Jr., Sauer, F., Hu, B., Bascle, B., Geiger, B., Wenzel, F., Rohlfing, T., Brown, C. M., Bakos, R. S., Maciunas, R. J., AND Bani-hashemi, A. 2001. Augmented reality visualization of brain structures with stereo and kinetic depth cues: System description and initial evaluation with head phantom. In Medical Imaging 2001: Visualization, Display, and Image-Guided Procedures, Proc. SPIE 4319, Society of Photo-Optical Instrumentation Engineers, 445-456.

Nakayama, K., Shimojo, S., and Ramachandran, V. S. 1990. Transparency: relation to depth, subjective contours, luminance, and neon color spreading. Perception 19, 497-513.

Salah, Z., Bartz, D., AND Strasser, W. 2005. Illustrative rendering of segmented anatomical data. In In Proc. of Simulation und Visualisierung, 175-184.

SCHWARTZ, J. T., 2003. Test yourself for stereo blindness. http://www.settheory.com/stereo_blindness_test.html.

Sielhorst, T., Bichlmeier, C., Heining, S. M., AND NAVAB, N. 2006. Depth perception - a major issue in medical ar: Evaluation study by twenty surgeons. In Proceedings of MICCAI 2006, LNCS 4190, 364-372.

Sielhorst, T., Feuerstein, M., And Navab, N. 2008. Advanced medical displays: A literature review of augmented reality. Journal of Display Technology 4, 4, 451-467.

TODD, J. T. 2004. The visual perception of $3 \mathrm{~d}$ shape. TRENDS in Cognitive Sciences 8, 3, 115-121.

Tsirlin, I., Allison, R. S., AND Wilcox, L. M. 2008. Stereoscopic transparency: Constraints on the perception of multiple surfaces. Journal of Vision 8, 5 (May), 1-10.

WAng, C., Frimmel, H., And Smedby, Ö. 2011. Level-set based vessel segmentation accelerated with periodic monotonic speed function. In Medical Imaging 2011: Image Processing, Proc. SPIE 7962, Society of Photo-Optical Instrumentation Engineers.

Zheng, L., WU, Y., AND MA, K. 2013. Perceptually based depthordering enhancement for direct volume rendering. IEEE Transactions on Visualization and Computer Graphics 19, 3, 446-459. 